\title{
Image-Guided Convection-Enhanced Delivery Platform in the Treatment of Neurological Diseases
}

\author{
Massimo S. Fiandaca, ${ }^{* \dagger}$ John R. Forsayeth, ${ }^{*}$ Peter J. Dickinson, ${ }^{*}$ and Krystof S. Bankiewicz* \\ *Department of Neurological Surgery, Laboratory of Molecular Therapeutics, University of California San Francisco, San \\ Francisco, California 94103; 'Department of Neurosurgery, LifeBridge Health Brain \& Spine Institute, Baltimore, Maryland \\ 21209; and ${ }^{\ddagger}$ Department of Surgical and Radiological Sciences, School of Veterinary Medicine, University of California-Davis, \\ Davis, California 95616
}

\begin{abstract}
Summary: Convection-enhanced delivery (CED) of substances within the human brain is becoming a more frequent experimental treatment option in the management of brain tumors, and more recently in phase 1 trials for gene therapy in Parkinson's disease (PD). Benefits of this intracranial drugtransfer technology include a more efficient delivery of large volumes of therapeutic agent to the target region when compared with more standard delivery approaches (i.e., biopolymers, local infusion). In this article, we describe specific technical modifications we have made to the CED process to make it more effective. For example, we developed a reflux-resistant infusion cannula that allows increased infusion rates to be used. We also describe our efforts to visualize the CED process in vivo, using liposomal nanotechnology and real-time intraoper-
\end{abstract}

ative MRI. In addition to carrying the MRI contrast agent, nanoliposomes also provide a standardized delivery vehicle for the convection of drugs to a specific brain-tissue volume. This technology provides an added level of assurance via visual confirmation of CED, allowing intraoperative alterations to the infusion if there is reflux or aberrant delivery. We propose that these specific modifications to the CED technology will improve efficacy by documenting and standardizing the treatment-volume delivery. Furthermore, we believe that this image-guided CED platform can be used in other translational neuroscience efforts, with eventual clinical application beyond neuro-oncology and PD. Key Words: Convection-enhanced delivery, MRI, infusion cannula, CNS, gene transfer, liposomes, brain neoplasm, Parkinson's disease, trophic factors.

\section{INTRODUCTION}

Delivery of therapeutic agents into the brain has been an ongoing challenge to clinicians and scientists for many years. Intravascular administrations of therapeutic agents have been confounded by the efficiency of the blood-brain barrier (BBB) $)^{1}$ that allows entrance of molecules based on size, lipid solubility and charge, ${ }^{2,3}$ severely limiting entry of charged small molecules or larger compounds such as proteins or viral particles. Disruption of the BBB has been used clinically to temporarily broach this natural barrier between the brain and the vascular system, ${ }^{2,4}$ allowing a greater quantity and variety of intravascular therapeutic agents to access the brain parenchyma, including monoclonal antibodies and radio-immunoconjugates. ${ }^{5,6}$ This technology has been

Address correspondence and reprint requests to: Krystof S. Bankiewicz, MD, PhD, Department of Neurological Surgery, MCB226, 1855 Folsom Street, University of California San Francisco, San Francisco, CA 94103-0555. E-mail: krystof.bankiewicz@ucsf.edu. successful in the treatment of many neuro-oncologic conditions in a number of centers, ${ }^{7}$ and especially with primary central nervous system (CNS) lymphoma. ${ }^{8} \mathrm{Un}$ fortunately, despite selective opening of unilateral or bilateral vertebrobasilar or carotid cerebrovascular distributions via the arterial approach, controlling the region of treatment through this technique remains complicated and limited to a major vascular distribution. In addition, repeated treatments add to patient discomfort and potential morbidity, ultimately narrowing the clinical use of osmotic BBB disruption therapy.

Neurosurgeons have been circumventing the BBB for decades via more direct approaches to the brain parenchyma and cerebrospinal fluid spaces. In many cases of brain tumor, therapeutic materials have been placed within the adjacent brain or tumor bed after resection. ${ }^{9}$ Various catheters/cannulae have been stereotactically placed into the brain or CSF spaces for more precise delivery of therapeutic compounds in cases of tumor, ${ }^{10-12}$ and more recently PD. ${ }^{13,14}$ Biopolymer tech- 
nology has allowed chemotherapeutic agents to be placed and gradually released at the brain surface subsequent to tumor resection, providing higher local concentrations than possible via systemic delivery, without the associated systemic toxicity. ${ }^{15-17}$ Depending on the biopolymer composition, drugs can be delivered in a controlled manner for a finite period of time. Unfortunately, reoperation is required for re-dosing of the patient with these biopolymers, and controversy remains over cost and associated morbidities. Catheter techniques allow repeated dosing at the specific site of catheter placement, usually without significant discomfort or morbidity for the patient, especially when coupled with subcutaneous reservoirs or mini-pumps. ${ }^{18,19}$ Through these techniques, the BBB is bypassed, allowing the specific therapeutic agent direct access to the brain parenchyma and CSF. Unfortunately, because all of these modalities depend on diffusion of their treatment materials from the source (i.e., biopolymer, catheter openings), as previously noted in preclinical studies, the diffusion distance is limited to the immediate (several $\mathrm{mm}$ ) surrounding brain volume. ${ }^{17}$ Diffusion is linked directly to the concentration gradient and diffusion coefficient of the molecule, with molecular size having an inverse relationship with diffusion..$^{20-22}$ Limits to diffusion of more than several millimeters for low molecular weight compounds include capillary clearance and, in some cases, metabolism. ${ }^{23-25}$ To date, treatments using these diffusion technologies remain difficult to standardize due to lack of control of the diffusion and the associated heterogeneity of distribution of the therapeutic substances due to some of the factors previously described. In addition, the extent of diffusion in vivo is difficult to confirm, making any treatment standardization and comparisons difficult at best, and usually impossible.

Convection-enhanced delivery (CED) technology offers the clinician a more specific option in delivering therapeutic agents to a larger and more consistent treatment volume than the standard diffusion-based technologies. CED uses bulk flow of the therapeutic material through the extracellular space of the tissue. ${ }^{26}$ Bulk flow distributes therapeutic agents homogeneously within a controlled brain volume, regardless of molecular size, with a steep concentration drop at the advancing margin of the bulk flow. This fact opens the door to convection of viral particles (for gene therapy) and large macromolecules (e.g., growth factors) into the brain, in addition to other substances. The volume in which a particle (i.e., drug, virus or growth factor) distributes with CED in the brain is primarily the function of infusion rate and specific tissue characteristics. These brain tissue characteristics and CED have been modeled in animals, ${ }^{27,28}$ and have recently been evaluated in human brain tumor trials. ${ }^{12,29}$ CED infusion information is now commercially available for neuro-oncology as a treatment software al- gorithm developed by BrainLAB through their iPlan Flow software (www.brainlab.com) (BrainLAB AG, Feldkirchen, Germany), which was approved by the Food and Drug Administration. This program essentially allows the planning of drug (or therapeutic material) delivery into the human brain based on the MRI data and brain tissue characteristics of the individual patient. Treatment of specific tumor volumes can be preplanned in three-dimension ${ }^{28}$ (based on various infusion catheter positions) in an effort to maximize tumor coverage with the least number of catheters, in a manner similar to isodose planning for stereotactic radiosurgery. ${ }^{30} \mathrm{Al}$ though this computer technology allows very reliable planning of treatment based on accumulated brain MRI data, it does not give the neurosurgeon real-time visualization of the CED infusion process and has yet to be fully, clinically validated in real-time with a co-convected tracer. Visualizing the CED process has recently been retrospectively carried out in patients with gliomas, using radiolabelled albumin coinfused with a cytotoxin, ${ }^{31,32}$ and using gadolinium diethylene triamine pentaacetic acid (DTPA) coinfusion for brainstem lesions. ${ }^{33}$ Although the former studies ${ }^{31,32}$ did not allow real-time adjustment of the CED procedure and relied on a delayed analysis of nuclear medicine imaging studies overlayed on MRI images, the latter study ${ }^{33}$ documented the potential for real-time MRI imaging in clinical situations in which the extent of delivery and possibility of leakage are most critical. Despite some continued limitations, these CED planning techniques are being used as part of our experimental neuro-oncologic studies ${ }^{34}$ and also in clinical neurosurgery. ${ }^{35}$

A common problem with infusions of a drug into the brain, both with injection techniques and CED, has been reflux along the catheter/cannula track. This is critical in CED because reflux prevents continued pressure in the extracellular space that drives the bulk fluid flow away from the catheter that helps determine the eventual distribution volume. ${ }^{26}$ Once reflux develops (FIG. 1) around an infusion or CED catheter/cannula, there is a plateau to the volume of distribution $(\mathrm{Vd})$ while the volume infused (Vi) increases, and the difference is lost as reflux. This reflux and lack of increase in $\mathrm{Vd}$ indicates preferential flow of additional $\mathrm{Vi}$ along the catheter/cannula tract, away from the treatment region (e.g., tumor, subthalamic nucleus, or striatum) and usually into the CSF. This is especially important in trying to avoid local or systemic toxicities associated with infused materials and to help standardize treatment protocols based on infused volumes. In a recent clinical study sponsored by Amgen (Thousand Oaks, CA), high doses (15 $\mu \mathrm{g} /$ day) of glialderived neurotrophic factor (GDNF) were chronically infused into the putamen of 17 PD patients for at least 6 months. ${ }^{36}$ Although efficacy has not been shown for this treatment protocol, 3 of these patients developed anti- 


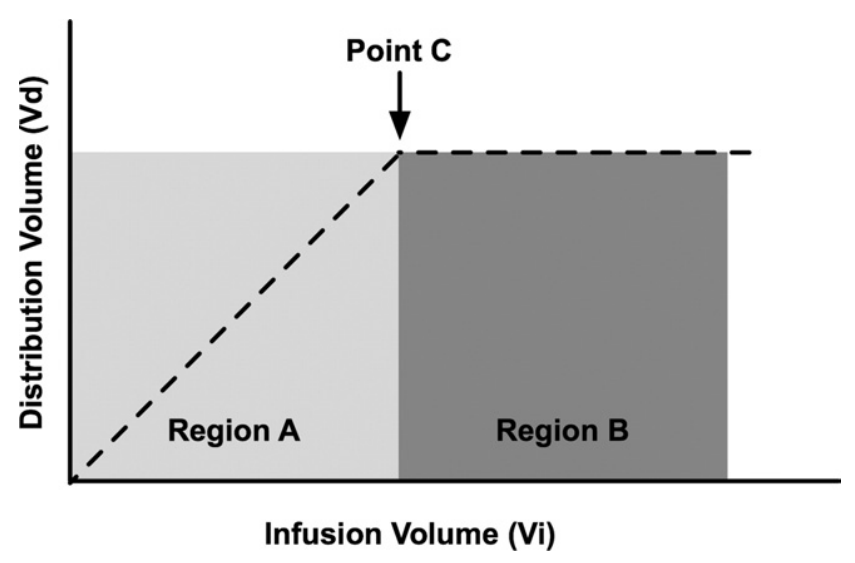

FIG 1. Schematic representation of changes in volume of distribution (Vd) and volume infused (Vi) during convection-enhanced delivery (CED), with and without reflux. In this graph, changes in $\mathrm{Vd}$ and $\mathrm{Vi}$ are represented by the dotted lines. Region A represents a normal CED pattern starting at low $\mathrm{Vi}(\mu \mathrm{L})$, and $\mathrm{Vd}$ $(\mu \mathrm{L})$ increases linearly in proportion to the $\mathrm{Vi}$ with the slope $\mathrm{Vd} / \mathrm{Vi}$, characteristic of the infusate and the rate of infusion ( $\mu \mathrm{L} / \mathrm{min})$. Point $\mathrm{C}$ represents the volume at which reflux begins, with the slope of region $B$ approaching zero. Region $B$ represents the region of reflux, where despite increases in $\mathrm{Vi}, \mathrm{Vd}$ does not increase.

bodies to the GDNF, probably due to systemic exposure of the infused protein through reflux into the CSF and the venous system. Although it is clear that many unresolved issues exist in this and other infusion studies, major areas of potential technical refinement include: 1) optimal drug dose, 2) infusion volume, 3) infusion rate, 4) catheter design and 5) prevention of reflux.

One of our goals has been to more clearly define and solve the technical problems related to CED infusion therapies that would eventually open the technology to applicability in a wide variety of clinical situations. With our concentration in neuro-oncology and PD, our CED efforts progressed in both of these areas and have recently been translated to human clinical trials (see description as follows).

CED has been the method of choice for therapeutic agent delivery in our laboratory for many years. Through studies in rats, dogs and nonhuman primates, it became obvious that CED offered the capabilities that we were looking for in scaling up our experimental treatment volumes to approach those in the human brain. Determination of appropriate drug or therapeutic agent doses remains overly complex for this discussion and requires individual assessment and clinical trials for more definitive answers. Infusion volumes with CED are usually determined by the proposed clinical target size (i.e., tumor volume or brain target volume). The relationship between the $\mathrm{Vi}$ and $\mathrm{Vd}$ is linear, but the slope $(\mathrm{Vd} / \mathrm{Vi})$ of the curve is dependent on structural properties of the tissue, such as degree of myelination ${ }^{37}$ or the characteristics of the infusate. ${ }^{26,37}$ As previously noted (see previous BrainLAB discussion), specific tissue data to pre- dict optimal infusion volumes and other measurements (e.g., catheter location) to cover the proposed Vd have been determined and allow clinical planning prior to CED infusion. Vd can be enhanced for some therapeutic agents (without increasing $\mathrm{Vi}$ ) through the manipulation of physiologic measurements (i.e, blood pressure/heart rate $)^{38}$ and the use of heparin coinfusion. ${ }^{39}$

An optimal infusion rate for CED has been elusive because it is often limited by the development of reflux along the cannula track. In most cases, the optimal infusion rate is that which allows the delivery of the therapeutic volume over the least amount of time without associated reflux. With all catheters/cannulas, reflux can be induced if the infusion rate is too high. The larger the catheter diameter, the more easily reflux can be induced. $\mathrm{We},{ }^{40}$ along with others, ${ }^{27,41,42}$ have documented that CED infusion flow rates less than $0.5 \mu \mathrm{L} / \mathrm{min}$ may be necessary to prevent reflux in catheters up to $0.6 \mathrm{~mm}$ (outer diameter $[\mathrm{OD}]$ ). However, in a recent phase III trial of transferrin-CRM107 (Xenova Biomedix Ltd, Guilford, Surrey, UK) in glioblastoma, investigators used a ventriculo-peritoneal shunt catheter ( $2.5 \mathrm{~mm} \mathrm{OD})$ at a flow rate of $3.3 \mu \mathrm{L} / \mathrm{min}$ (unpublished data). In this clinical study, one could predict a significant reflux of transferrin-CRM107 into the subarachnoid space and outside the brain parenchyma, based on the prior experimental data. ${ }^{27,40-42}$ Communication with a trial coordinator confirmed that this has indeed been a suspected problem (S. Kunwar, personal communication). A similar issue was found with CED used to deliver IL13PE38QQR immunotoxin (Neopharm, Waukegan, IL) in glioblastoma. A similar delivery catheter (1.4 mm OD) was used (S. Kunwar, personal communication) and MRI signal changes were noted in the catheter tracks after infusion ${ }^{11}$ in all 14 patients in the phase I study, possibly due to reflux.

Recently, ${ }^{40}$ we have increased the potential CED infusion rate to $5 \mu \mathrm{L} / \mathrm{min}$ (10-fold increase) without reflux with an innovative cannula design. The new reflux-resistant, step design cannula features a 27 -gauge $(0.2 \mathrm{~mm})$ needle with a glued-in, internal silica tubing (the differing diameters making the step) that extends beyond the end of the needle by up to $10 \mathrm{~mm}$ in humans and $5 \mathrm{~mm}$ or less in nonhuman primates or canines. The silica has an OD of $168 \mu \mathrm{m}$ and an inner diameter of $102 \mu \mathrm{m}$. The smaller cannula design avoids tissue trauma during insertion. Pushing flow rates significantly above $5 \mu \mathrm{L} / \mathrm{min}$, however, can induce reflux even with this catheter (see FIG. 2), and can increase the likelihood of causing local brain tissue damage. ${ }^{40}$ This design is being used in a phase I clinical trial at the University of California, San Francisco to deliver AAV2-hAADC (adeno-associated virus 2 carrying human aromatic amino acid decarboxylase gene) in 15 patients with moderate to severe PD (Eberling et al., manuscript submitted for publication). 

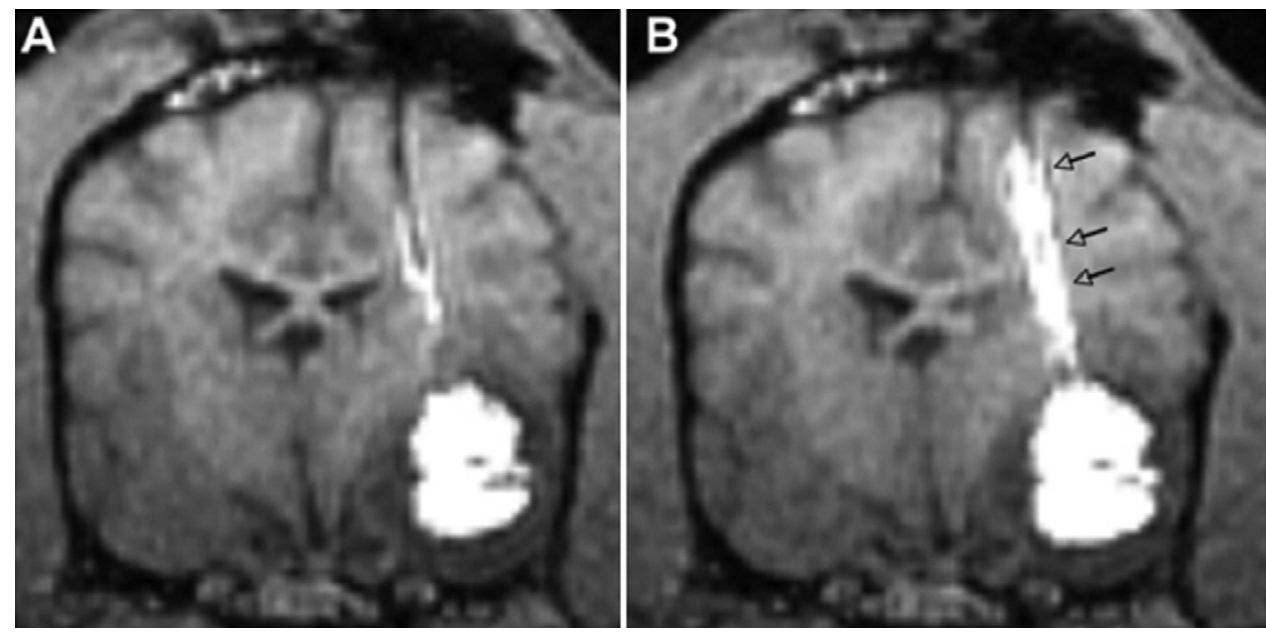

FIG 2. Reflux at high infusion rates: $1.5 \mathrm{~T}$ MRI T1-weighted spoiled gradient recalled intraoperative images of real-time convectionenhanced delivery (CED) in the treatment of a case of spontaneous canine piriform lobe tumor. CED of Gadoteridol (Prohance)-labeled neutral nanoliposomes into the canine tumor through the reflux-resistant cannula gives very little reflux at $5 \mu \mathrm{L} / \mathrm{min}(\mathrm{A})$, but it gives quite significant reflux along the cannula (see arrowheads) at $8 \mu \mathrm{L} / \mathrm{min}(\mathrm{B})$.

Since CED monitored with MRI imaging was initially described in rodents, ${ }^{43}$ our laboratory has advanced the concept of real-time imaging of the CED process in vivo by co-infusing Gadoteridol (Prohance)-labeled (Bracco Diagnostic Inc., Princeton, NJ) neutral nanoliposomes (GDL) that are convected along with the therapeutic agent (e.g., AAV2, GDNF or CPT-11) in experimental animals, ${ }^{28,34}$ allowing visualization of the convection process. Neutral nanoliposomes not only carry the MRI contrast agent in this case, but also enhance the $\mathrm{Vd}$ of the convected material carried within the nanoliposome (i.e., drug, neurotrophic factor, viral particle) by preventing early binding of the material to the extracellular matrix or cellular lipid membranes. ${ }^{37}$ We have shown that the $\mathrm{Vd}$ of the various agents carried within the neutral nanoliposomes was identical, making the nanoliposome an excellent carrier for therapeutic agents, and providing a consistent Vd profile. Drug encapsulation within liposomes allows a better $\mathrm{Vd}$ of lipophilic agents (i.e., $\beta$-chloro-nitrosourea, carmustine [Ben Venue Laboratories, Inc., Bedford, $\mathrm{OH}]$ and others), preventing nonspecific toxicities at local diffusion distances, while providing prolonged contact time of effective doses within significant brain tumor volumes. ${ }^{44}$ GDL delivery of gene therapy products has shown excellent correlation between imaging and histochemical staining for the transgene (Bankiewicz et al., manuscript in preparation), providing confidence in the co-distribution of MRI contrast agent and convected therapeutic material. As previously noted, gadolinium DTPA tracer has also been used safely in a limited number of patients with brainstem lesions treated with CED. ${ }^{33}$

Although image-guided CED requires the added costs of an intraoperative MRI scanner, and potential morbidity associated with the GDL (or other contrast agent) delivered to the brain, the time-sensitive information that this provides intraoperatively for the treating neurosurgeon is immeasur- able. This is critical because we have recently noted that despite using our reflux-resistant cannula and flow rates less than $5 \mu \mathrm{L} / \mathrm{min}$, we documented reflux in almost $20 \%$ of the infusion tracks in both canine and nonhuman primate experimental subjects undergoing image-guided CED with GDL (Varenika et al., manuscript in review) (FIG. 2). This real-time information allows for adjustments in treatment that could be made "on the fly" by the neurosurgeon. In some cases, reducing the infusion rate can allow effective CED to continue without reflux. More drastic changes would include adjusting the position of the infusion catheter or placing an additional catheter to cover the planned treatment volume. Without the ability to monitor CED in realtime, therefore, reflux (and $\mathrm{Vd}$ ) cannot be accurately documented; thus real-time optimization is not possible, and treatment volumes will remain too variable to allow valid comparisons between patients in therapeutic trials. Treatment volumes may be even more critical when comparing PD patients who undergo CED gene therapy or neurotrophic factor therapy of the putamen, subthalamic nucleus, or substantia nigra. Real-time imaging and a liposomal carrier would allow visualization of the convected volume and a more reproducible $\mathrm{Vd}$, despite differences in the selected therapeutic agent.

It is this wide-ranging applicability in the precise delivery of drugs, macromolecules and viral particles that encourages us to continue the development of this CED technology. What is abundantly clear to us at this stage is that the many optimizations of CED that we have worked out in animal experiments and have translated to human trials will be advanced even further through the use of a real-time, image-guided CED platform to more precisely deliver therapeutic materials into the brain. We also predict the use of this technology beyond neuro-oncology and PD, for the treatment of other neurological diseases. 
Acknowledgments: We would like to thank Dr. Sandeep Kunwar for communicating unpublished data. Our studies have been supported by grants to Krystof S. Bankiewicz by a Specialized Programs of Research Excellence grant from the National Cancer Institute and grants from the National Institutes of Health.

\section{REFERENCES}

1. Oldendorf WH. Lipid solubility and drug penetration of the blood brain barrier. Proc Soc Exp Biol Med 1974;147:813-815.

2. Neuwelt EA. Mechanisms of disease: the blood-brain barrier. Neurosurgery 2004;54:131-140; discussion 141-132.

3. Motl S, Zhuang Y, Waters CM, Stewart CF. Pharmacokinetic considerations in the treatment of CNS tumours. Clin Pharmacokinet 2006;45:871-903.

4. Kroll RA, Neuwelt EA. Outwitting the blood-brain barrier for therapeutic purposes: osmotic opening and other means. Neurosurgery 1998;42:1083-1099; discussion 1099-1100.

5. Feugier P, Van Hoof A, Sebban C, et al. Long-term results of the R-CHOP study in the treatment of elderly patients with diffuse large B-cell lymphoma: a study by the Groupe d'Etude des Lymphomes de l'Adulte. J Clin Oncol 2005;23:4117-4126.

6. Nowakowski GS, Witzig TE. Radioimmunotherapy for B-cell nonHodgkin lymphoma. Clin Adv Hematol Oncol 2006;4:225-231.

7. Doolittle ND, Miner ME, Hall WA, et al. Safety and efficacy of a multicenter study using intraarterial chemotherapy in conjunction with osmotic opening of the blood-brain barrier for the treatment of patients with malignant brain tumors. Cancer 2000;88:637-647.

8. Jahnke K, Doolittle ND, Muldoon LL, Neuwelt EA. Implications of the blood-brain barrier in primary central nervous system lymphoma. Neurosurg Focus 2006;21:E11.

9. Brem H, Mahaley MS, Jr., Vick NA, et al. Interstitial chemotherapy with drug polymer implants for the treatment of recurrent gliomas. J Neurosurg 1991;74:441-446.

10. Mardor Y, Roth Y, Lidar Z, et al. Monitoring response to convection-enhanced taxol delivery in brain tumor patients using diffusion-weighted magnetic resonance imaging. Cancer Res 2001;61: 4971-4973.

11. Kunwar S. Convection enhanced delivery of IL13-PE38QQR for treatment of recurrent malignant glioma: presentation of interim findings from ongoing phase 1 studies. Acta Neurochir Suppl 2003;88:105-111.

12. Lidar Z, Mardor Y, Jonas T, et al. Convection-enhanced delivery of paclitaxel for the treatment of recurrent malignant glioma: a phase I/II clinical study. J Neurosurg 2004;100:472-479.

13. Gill SS, Patel NK, Hotton GR, et al. Direct brain infusion of glial cell line-derived neurotrophic factor in Parkinson disease. Nat Med 2003;9:589-595.

14. Patel NK, Bunnage M, Plaha P, Svendsen CN, Heywood P, Gill SS. Intraputamenal infusion of glial cell line-derived neurotrophic factor in PD: a two-year outcome study. Ann Neurol 2005;57:298-302.

15. Brem $\mathrm{H}$, Gabikian P. Biodegradable polymer implants to treat brain tumors. J Control Release 2001;74:63-67.

16. Fleming AB, Saltzman WM. Pharmacokinetics of the carmustine implant. Clin Pharmacokinet 2002;41:403-419.

17. Gallia GL, Brem S, Brem H. Local treatment of malignant brain tumors using implantable chemotherapeutic polymers. J Natl Compr Canc Netw 2005;3:721-728.

18. Bakhshi S, North RB. Implantable pumps for drug delivery to the brain. J Neurooncol 1995;26:133-139.

19. Chandler WF, Greenberg HS, Ensminger WD, et al. Use of implantable pump systems for intraarterial, intraventricular and intratumoral treatment of malignant brain tumors. Ann N Y Acad Sci 1988;531:206-212.

20. Schantz EJ, Lauffer MA. Diffusion measurements in agar gel. Biochemistry 1962;1:658-663.

21. Jain RK. Transport of molecules in the tumor interstitium: a review. Cancer Res 1987;47:3039-3051.

22. Jain RK. Delivery of novel therapeutic agents in tumors: physiological barriers and strategies. J Natl Cancer Inst 1989;81:570576.

23. Kroin JS, Penn RD. Intracerebral chemotherapy: chronic microinfusion of cisplatin. Neurosurgery 1982;10:349-354.
24. Sendelbeck SL, Urquhart J. Spatial distribution of dopamine, methotrexate and antipyrine during continuous intracerebral microperfusion. Brain Res 1985;328:251-258.

25. Morrison PF, Dedrick RL. Transport of cisplatin in rat brain following microinfusion: an analysis. J Pharm Sci 1986;75:120-128.

26. Bobo RH, Laske DW, Akbasak A, Morrison PF, Dedrick RL, Oldfield EH. Convection-enhanced delivery of macromolecules in the brain. Proc Natl Acad Sci U S A 1994;91:2076-2080.

27. Degen JW, Walbridge S, Vortmeyer AO, Oldfield EH, Lonser RR. Safety and efficacy of convection-enhanced delivery of gemcitabine or carboplatin in a malignant glioma model in rats. J Neurosurg 2003;99:893-898.

28. Krauze MT, Forsayeth J, Park JW, Bankiewicz KS. Real-time imaging and quantification of brain delivery of liposomes. Pharm Res 2006;23:2493-2504.

29. Kioi M, Husain SR, Croteau D, Kunwar S, Puri RK. Convectionenhanced delivery of interleukin-13 receptor-directed cytotoxin for malignant glioma therapy. Technol Cancer Res Treat 2006;5:239250 .

30. Papagiannis P, Karaiskos P, Kozicki M, et al. Three-dimensional dose verification of the clinical application of gamma knife stereotactic radiosurgery using polymer gel and MRI. Phys Med Biol 2005;50:1979-1990.

31. Sampson JH, Raghavan R, Brady ML, et al. Clinical utility of a patient-specific algorithm for simulating intracerebral drug infusions. Neuro Oncol 2007;9:343-353.

32. Sampson JH, Brady ML, Petry NA, et al. Intracerebral infusate distribution by convection-enhanced delivery in humans with malignant gliomas: descriptive effect of target anatomy and catheter positioning. Neurosurgery 2007;60(2 Suppl 1):89-98.

33. Lonser RR, Warren KE, Butman JA, et al. Real-time image-guided direct convective perfusion of intrinsic brainstem lesions. Technical note. J Neurosurg 2007;107:190-197.

34. Noble CO, Krauze MT, Drummond DC, et al. Novel nanoliposomal CPT-11 infused by convection-enhanced delivery in intracranial tumors: pharmacology and efficacy. Cancer Res 2006;66:2801-2806.

35. Kunwar S, Chang SM, Prados MD, et al. Safety of intraparenchymal convection-enhanced delivery of cintredekin besudotox in early-phase studies. Neurosurg Focus 2006;20:E15.

36. Lang AE, Gill S, Patel NK, et al. Randomized controlled trial of intraputamenal glial cell line-derived neurotrophic factor infusion in Parkinson disease. Ann Neurol 2006;59:459-466.

37. Saito R, Krauze MT, Bringas JR, et al. Gadolinium-loaded liposomes allow for real-time magnetic resonance imaging of convection-enhanced delivery in the primate brain. Exp Neurol 2005;196: 381-389.

38. Hadaczek P, Mirek H, Tamas L, et al. Perivascular pump" driven by arterial pulsation is a powerful mechanism for the distribution of therapeutic molecules within the brain. Mol Ther 2006;14:69-71.

39. Hamilton JF, Morrison PF, Chen MY, et al. Heparin coinfusion during convection-enhanced delivery (CED) increases the distribution of the glial-derived neurotrophic factor (GDNF) ligand family in rat striatum and enhances the pharmacological activity of neurturin. Exp Neurol 2001;168:155-161.

40. Krauze MT, Saito R, Noble C, et al. Reflux-free cannula for convection-enhanced high-speed delivery of therapeutic agents. J Neurosurg 2005;103:923-929.

41. Lonser RR, Walbridge S, Garmestani K, et al. Successful and safe perfusion of the primate brainstem: in vivo magnetic resonance imaging of macromolecular distribution during infusion. J Neurosurg 2002;97:905-913.

42. Morrison PF, Chen MY, Chadwick RS, Lonser RR, Oldfield EH. Focal delivery during direct infusion to brain: role of flow rate, catheter diameter, and tissue mechanics. Am J Physiol 1999;277: $1218-1229$.

43. Mardor Y, Rahav O, Zauberman Y, et al. Convection-enhanced drug delivery: increased efficacy and magnetic resonance image monitoring. Cancer Res 2005;65:6858-6863.

44. Saito R, Bringas JR, McKnight TR, et al. Distribution of liposomes into brain and rat brain tumor models by convection-enhanced delivery monitored with magnetic resonance imaging. Cancer Res 2004;64:2572-2579. 